\title{
ESTATURA, DIFERENCIAS REGIONALES Y SOCIALES Y NIVELES DE VIDA EN ESPAÑA (1893-1954)
}

\author{
GLORIA QUIROGA VALLE *
}

Universidad Carlos III de Madrid

\section{RESUMEN}

Este artículo muestra un estudio antropométrico de los reclutas españoles entre 1893-1954, con el fin de explorar una aproximación a los niveles de bienestar en España. Se señala la importancia de la estatura como indicador del nivel nutricional y epidemiológico, así como sus determinantes. Basándose en las Hojas de Filiación de los reclutas españoles, se presentan las series de estatura media a nivel nacional, regional y socioprofesional. Los resultados indican cambios y diferencias en el bienestar de los españoles, una estrecha correlación entre estatura, renta, condiciones sanitarias, y cómo la estatura puede ser un indicador de la evolución del grado de desigualdad en las condiciones materiales de vida, mostrando para España un diferente comportamiento espacial y sectorial.

\section{ABSTRACT}

This article applies an anthropometric study to Spanish conscripts between 1893-1954 in order to explore a proxy for living standards in Spain. The first part of the article introduces the importance of height as an indicator of levels of nutrition and epidemiological enviroment, and its determinants. The second part uses the conscripts files to calculate average heights at a national and regional level and by socio-professional categories. This section shows changes and differences in Spanish living standards. A second finding is the strong correlation between height, income and sanitary conditions. Finally, height can be used as an indicator of the evolution of the degree of inequality in material living conditions which in the case of Spain shows a divergent behaviour between regions and socio-professional categories.

* La autora agradece todas las sugerencias de los asistentes al seminario «Nuevas interpretaciones en la Historia Económica de España» (UIMP.Cuenca, junio, 2000). Asimismo, los comentarios recibidos por parte de Jordi Palafox, Albert Carreras, Sebastián Coll, Stefan Houpt y los evaluadores anónimos han contribuido a mejorar el resultado final. No obstante, toda la responsabilidad sobre los errores cometidos es exclusivamente mía. 


\section{INTRODUCCIÓN}

La atención prestada por la Historia Económica a los niveles de vida de las poblaciones en el pasado ha estado centrada habitualmente en los indicadores tradicionales, especialmente renta por habitante y salarios reales. No obstante, el uso de estos indicadores económicos plantea problemas tanto teóricos como empíricos relacionados con su construcción (bienes incluidos y excluidos, ponderaciones utilizadas, no contabilización de algunas utilidades y servicios intangibles, etc.), y con la disponibilidad de fuentes fiables para estas variables que sean a su vez representativas de todo el espectro social ${ }^{1}$.

Ante esta situación, los científicos sociales y los organismos internacionales han intentado crear indicadores de desarrollo más universales y fáciles de construir, sustituyendo así el concepto de nivel de vida por uno más amplio como es el de bienestar y recurriendo para ello al uso de nuevas variables ${ }^{2}$. De este conjunto de nuevas variables destacan las medidas antropométricas. En especial la estatura, que es un excelente indicador del estado nutricional, del nivel sanitario, del medio ambiente epidemiológico, del desgaste físico, y en definitiva de las condiciones materiales de vida para determinadas fases de desarrollo.

A la vez que la estatura contempla aspectos de las condiciones materiales de vida descuidados por los indicadores económicos, es posible que, a propósito de determinadas sociedades del pasado, englobe a estos últimos. El punto de partida de esta metodología es la idea de que el consumo de alimentos en siglos pasados era el mayor componente de gasto familiar, lo que convierte a la estatura posiblemente en el mejor indicador del nivel nutricional de una población, y estrechamente emparentado con la renta per cápita, siempre a propósito de ese tipo de economías ${ }^{3}$.

Además, los datos sobre estatura presentan otras ventajas, como son la abundancia y extensión sobre diferentes grupos socioeconómicos y áreas

\footnotetext{
1 Véase Coll y Komlos (1998).

${ }^{2}$ A modo de ejemplo, véase Nordhaus y Tobin (1973). Una revisión del tema aparece en Offer (2000).

3 Durante la etapa comprendida entre los siglos xV y xvil, la mayor parte de la sociedad («la masa de la pobreza») gastaba entre el 70 y el 80 por 100 de sus ingresos en su alimentación, que no dejaba de ser escasa. Véase Cipolla (1992). En general, para cualquier tipo de economías, todos los factores que influyen en la estatura media de una población operan a través de la enfermedad y especialmente de la nutrición, que a su vez depende de los precios relativos de los alimentos, de los precios del resto de los bienes y especialmente, de la renta familiar y de la distribución del ingreso. Steckel (1992) y (1995).
} 
geográficas; a su vez, pueden ser utilizados para analizar mejoras en la nutrición y comportamientos socioeconómicos (especialmente en la productividad del trabajo y en el declive de la mortalidad) y, por último, pueden aportar información útil para conocer la evolución de la desigualdad, si no en la distribución del ingreso, al menos en las condiciones materiales de vida 4 .

Estas ventajas han permitido que el estudio de la evolución de esta variable se convierta en una línea de investigación muy fructífera en la historia económica internacional, denominada Historia Antropométrica, heredera de los estudios de economía del desarrollo ligados a organismos internacionales que proliferaron a partir de 1960. El objeto de estudio es la relación existente entre las medidas antropométricas y los niveles de vida, planteando el empleo de las primeras como indicador de los progresos en el nivel de vida a propósito de aquellos países en vías de desarrollo para los cuales se carece de información directa sobre el producto per cápita ${ }^{5}$. En realidad, estos estudios no hacían más que continuar, después de un largo paréntesis, la línea abierta por los primeros investigadores de la ciencia estadística a comienzos del siglo $\mathrm{xIX}$ al estudiar las relaciones existentes entre estatura, mortalidad y condiciones materiales de vida ${ }^{6}$.

Los historiadores económicos siguieron pronto el ejemplo, esta vez a propósito de la época preindustrial, para la cual tampoco existen series que proporcionen información sobre los niveles y las condiciones materiales de vida, y a menudo tampoco la información de base para reconstruirlas con garantías de fiabilidad. El impulso fundamental vino dado en la década de los ochenta por Fogel y sus colaboradores que empezaron a trabajar en diferentes países y para diferentes épocas y grupos sociales siguiendo esta metodología. Así, destacan los estudios llevados a cabo para el Imperio Austro-húngaro, Inglaterra, Irlanda, Suecia y Estados Unidos, que muestran, primero, la existencia de ciclos en la estatura física, dependiendo su evolución de factores medioambientales y alimenticios; y segundo, la

4 La estatura es mejor indicador cuanto más atrasada es la sociedad objeto de estudio pero una vez que se ha sobrepasado este nivel, la estatura va perdiendo capacidad de reflejo de bienestar. Obviamente, la estatura no puede seguir aumentando al mismo ritmo que lo hace la renta ni indefinidamente.

5 Eveleth y Tanner (1979).

- Véase entre otros Quetelet (1842) y (1871), Villermé (1829), Galton (1886/87), y en España, Oloriz (1896), Figuerola (?). 
divergencia, en ocasiones, de indicadores convencionales de bienestar económico y los indicadores antropométricos ${ }^{7}$.

Un inconveniente de la estatura es, sin embargo, que resulta un indicador referido a períodos de tiempo bastante más largos que los usuales en otros indicadores. En efecto, la estatura humana a una determinada edad refleja el impacto acumulativo nutricional neto, es decir, la diferencia entre los nutrientes ingeridos y el desgaste energético producido por el mantenimiento del metabolismo basal, por el trabajo y la actividad física y por la enfermedad, desde la etapa uterina hasta el período en el cual el crecimiento físico se detiene. Este momento depende de cuándo se produzca el «estirón» adolescente.

$\mathrm{El}$ crecimiento humano sigue un patrón bien definido, denominado curva de crecimiento, que acusa mayor velocidad durante los dos primeros años de vida, declive durante la infancia, y crecimiento rápido durante la adolescencia. Por tanto, la sensibilidad del crecimiento a la malnutrición o a la enfermedad depende de la edad en la que éstas actúan.

No obstante, el patrón reflejado por el crecimiento parece ser causa de las condiciones ambientales, que determinan, por ejemplo, la edad a la que se produce el estirón adolescente o la edad a la que se detiene el crecimiento. La literatura médica refleja que una malnutrición y/o unas condiciones ambientales desfavorables en los primeros años de vida pueden ser compensadas a lo largo de la infancia, mientras que si ésta ocurre en la adolescencia, se provocará un retraso en el crecimiento y en la maduración ósea inhibiendo el crecimiento ${ }^{8}$. En este caso, cuando el crecimiento se produzca va a ser menor y no existen posteriormente «oportunidades de recuperación» para conseguir la estatura final potencial, puesto que el final de la etapa de crecimiento coincide con el final de la adolescencia, y el único mecanismo de defensa es retrasar el período del «estirón». Obviamente el retraso no puede ser indefinido.

Por todo lo expuesto se desprende que la adolescencia es el período más sensible al crecimiento y el más importante en la consecución de la estatura final y que la edad a la que ésta se produce (entre los doce años de la actualidad y los veinte de épocas pasadas) variará en función del nivel nutricional y las condiciones ambientales a las que los individuos estén expuestos, siguiendo el patrón de, a mejor alimentación y mejor

${ }^{7}$ Véase al respecto, entre otros, Komlos (1989), (1994) y (1995); Floud, Watcher y Gregory (1990); Nicholas y Steckel (1991); Sandberg y Steckel (1980); Fogel y Engerman (1982). Para un buen estado de la cuestión, véase Steckel (1995) y Martínez Carrión (1995).

${ }^{8}$ Wieringen (1978), Eveleth (1985). 
ambiente epidemiológico, el estirón adolescente se experimentará a edades más tempranas, y viceversa.

El objetivo de este artículo es utilizar las medidas antropométricas, en concreto la estatura, para arrojar información sobre la evolución durante la primera mitad del siglo $\mathrm{xx}$ del estado de la población española masculina desde los puntos de vista de la alimentación, sanidad, higiene, vivienda, etc., componentes todos éstos de las condiciones materiales de vida, para poder complementar y/o matizar a otros indicadores económicos. Aunque la metodología no es nueva para el caso español, ya que existe el trabajo pionero de Gómez Mendoza y Pérez Moreda o los realizados por Martínez Carrión para el caso murciano, la principal aportación de este artículo se basa en que por primera vez se presenta una serie continua de estatura para España y para todas sus regiones desde 1893 hasta 1954 extraída a partir de las Hojas de Filiación de los reclutas españoles y que permite enlazar con las estadísticas publicadas por el Ministerio de Defensa a partir de 1955 hasta nuestros días 9

\section{LAS HOJAS DE FILIACIÓN DE LOS RECLUTAS ESPAÑOLES}

A comienzos de la década de 1860 se estableció que cada municipio comenzara a recopilar sus propios Libros de Actas de Reclutamiento en los cuales debía anotarse información de todos y cada uno de los jóvenes que eran llamados a filas, mientras pasaban su reconocimiento médico. Simultáneamente, se rellenaba un formulario, llamado Hoja de Filiación, que constituía la primera pieza del expediente individual del mozo. En dicha hoja se registraban, entre los datos personales, su estatura. Afortunadamente, el Ejército ha conservado todos esos expedientes, centralizándolos en el Archivo General Militar Sección de Tropa, donde se encuentran unos dieciocho millones de expedientes militares con su Hoja de Filiación correspondiente, uno por cada mozo español que o bien fue excluido o bien cumplió su Servicio Militar en el Ejército de Tierra.

Debido a la abundancia de datos que existe en el Archivo, tuvimos que recurrir al muestreo para reducir la población estadística a una base de datos más manejable. Para realizar este muestreo se distinguieron dos

${ }^{9}$ Gómez Mendoza y Pérez Moreda (1985); Martínez Carrión (1985), (1994) y (1995); Martínez Carrión y Pérez Castejón (1998). El trabajo de Gómez Mendoza y Pérez Moreda (1985) sólo abarcaba desde 1912 a 1929. 
períodos: $1893-1910$ y $1911-1954^{10}$. La línea divisoria de ambos períodos viene dada porque en 1912 se instauró una nueva Ley de Reclutamiento por la que el Servicio Militar pasaba a ser obligatorio para todos los varones españoles ${ }^{11}$. Con anterioridad, figuras como la redención en metálico o el pago a un sustituto eran prácticas habituales entre las familias acomodadas, lo que implica que los expedientes anteriores a 1911 son una muestra sesgada hacia la presencia de varones procedentes de familias con ingresos bajos y menos representativa de la totalidad de la población española. Además, con anterioridad a 1911, sólo existen datos para algunas provincias españolas, mientras que a partir de esta fecha, se dispone de una base de datos prácticamente completa ${ }^{12}$. Por tanto, se utilizó una muestra aleatoria para seleccionar los expedientes posteriores a 1911, mientras que para el período anterior, el muestreo tuvo que ser modificado para evitar el sesgo.

Para el período 1893-1910, el método de muestreo no podía ser aleatorio, ya que se conocía la existencia de una sobre-representación de las clases agrarias y de ingresos bajos. Sin embargo, y sorprendentemente, observando los expedientes aparecieron ejemplos de estudiantes, profesionales o incluso rentistas, lo que permitió componer una muestra representativa con la condición de incluir a todos estos individuos. Así pues, la muestra se tomó recogiendo aleatoriamente miembros de los grupos de ingresos bajos y todos los individuos que pertenecieran a clases acomodadas, en igual número (veinte por grupo). A continuación, se ponderaron las medias correspondientes, usando como ponderación el número de habitantes de cada grupo atribuido por el censo de población más próximo, con el fin de calcular una media lo más representativa posible, dentro de todas estas limitaciones. Por ello, los resultados de este periodo deben ser tomados con cautela.

Entre 1911 y 1954, debido tanto a la propia organización del archivo como al deseo de poder establecer comparaciones regionales (se necesitaba una muestra que garantizara, para cada año, la presencia de un número suficiente de individuos pertenecientes a cada región), el muestreo fue

${ }^{10}$ Las razones de comenzar el estudio en 1893 obedecen a que es el primer año del que existen datos en el mencionado Archivo, y de terminar en 1954 es que a partir de esta fecha ya existen datos de estatura publicados por el Ministerio de Defensa.

$"$ Cuando se habla de año de reclutamiento, éste siempre es el anterior al de incorporación a filas; así, los tallados en 1911 ingresaron en el Ejército en 1912 y por ello fueron los primeros en sufrir el nuevo reglamento.

${ }_{12}$ Las excepciones son Cantabria, Murcia y Asturias cuyas series comienzan hacia 1930 , lagunas debidas a la Guerra Civil, durante la cual se destruyó mucha información. 
aleatorio polifásico resultando una muestra de 680 individuos por año, recogidos de 107 legajos. El objetivo era tener para cada una de las diecisiete Comunidades Autónomas un número de individuos no inferior a treinta (el límite convencional para considerar como normal la distribución muestral) y que fuera representativo de toda la población española, ya que ex post, la estatura media de cada provincia era ponderada por el número de mozos que formaban el reemplazo correspondiente ${ }^{13}$. La fiabilidad del muestreo queda garantizada por las leyes de la teoría del muestreo y todos los resultados muestran que los errores estándar de la media nacional estimada se encuentran en un orden de magnitud de unos $2,8 \mathrm{~mm}$, mientras que los errores típicos de las medias autonómicas estimadas muestran unos valores del orden de $9,4 \mathrm{~mm}^{14}$.

Otro factor que ha de tenerse en cuenta son los sucesivos cambios en la edad de reclutamiento, que aparecen recogidos en la legislación militar correspondiente ${ }^{15}$. Entre 1893 y 1907, la edad de talla de los reclutas pasó de diecinueve años a veintiún años. Asimismo, existieron múltiples alteraciones en la edad de reclutamiento producidas por la Guerra Civil y la posguerra ${ }^{16}$.

Antes de analizar los resultados, conviene hacer dos últimas precisiones: cuando se habla de un determinado año, siempre nos referimos al año de reclutamiento y no al de nacimiento (para calcular éste, sólo hay que restarle veintiuno a partir de 1907); y el criterio de clasificación de los reclutas es el lugar de residencia y no el de nacimiento. La elección de ambos criterios obedece, tal y como indica la literatura médica sobre el crecimiento, a que lo realmente relevante es donde el individuo pasó su adolescencia, la cual tenía más posibilidades de haber transcurrido en el lugar de reclutamiento que en el de nacimiento.

13 El hecho de utilizar la actual división administrativa en autonomías en lugar de las regiones históricas se debió a que el objetivo final del estudio es prolongar las series regionales hasta la actualidad y por ello es más coherente utilizar la actual regionalización.

${ }^{14}$ Para más información sobre su fiabilidad y la contrastación estadística según las leyes del muestreo, véase Quiroga (2001).

${ }^{15}$ Véase al respecto la Colección Legislativa del Ejército de cada año y Quiroga (2001)

i6 Con anterioridad a 1899, los jóvenes eran tallados con diecinueve años, pero en 1900 , se estableció en veinte y a partir de 1907 quedó fijada para todo el período en veintiún años. Durante la Guerra Civil y principio de la posguerra aparecieron distorsiones, y así los pertenecientes al reemplazo de 1938 fueron llamados con veinte años, y lo mismo es aplicable para los de 1941 a 1947, mientras que los de 1939 fueron tallados con veintidós. 


\section{EVOLUCIÓN DE LA ESTATURA EN ESPAÑA, 1893-1954}

El Gráfico 1 muestra la serie de la estatura media de los reclutas espanoles en medias móviles trienales para evitar las fluctuaciones espúreas que la muestra anual pueda presentar como consecuencia del error aleatorio de las muestras, pero al mismo tiempo, intentando no perder mucha información sobre la evolución de la variable ${ }^{17}$.

En el largo plazo, la serie de estatura media de los reclutas españoles muestra un incremento de tres centímetros a lo largo de sesenta años (exactamente $30,82 \mathrm{~mm}$ ). El crecimiento fue bastante modesto para un periodo de tiempo tan largo, especialmente si se tiene en cuenta que en los cuarenta y cinco años siguientes (1955-1999), el aumento ha sido de más de nueve centímetros $(95,21 \mathrm{~mm})^{18}$. Este escaso crecimiento contrasta con el experimentado por otros países europeos, como es el caso de Suecia que ve

\section{GRÁFICO 1}

Estatura media de los reclutas españoles, 1893-1954 (en mm). Medias móviles trienales

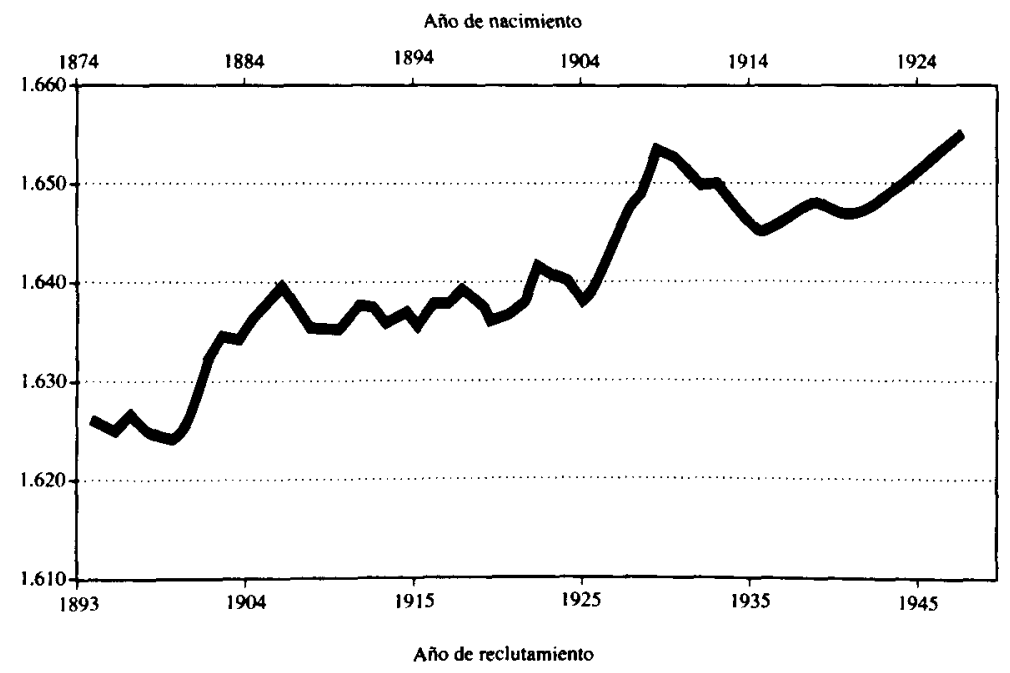

FUENTE: Archivo General Militar.

${ }^{17}$ No obstante, la serie anual aparece recogida en el Gráfico 2 (p. 184).

18 Quiroga (2001) a partir de la Estadística de Reclutamiento y Reemplazo, 1954-1999. 
cómo su estatura media aumentó en el mismo período de tiempo en más de cinco centímetros $(56,8 \mathrm{~mm})$ o los $63,8 \mathrm{~mm}$ de Holanda entre 1893 y 1945. Solamente, existe similitud con el caso italiano, que muestra un aumento de dos centímetros $(23,6 \mathrm{~mm})$ entre 1893 y $1942^{19}$.

Haciendo un examen más atento de la evolución de la serie de estatura, se pueden distinguir claramente seis fases. Así, existe un primer período (1894-1908) en el que aparece concentrado el mayor aumento de la estatura media (14,6 mm), seguido de una larga fase de estancamiento (1909-1928); a continuación (1929-1933), la serie de estatura experimenta su segundo mayor incremento $(13,29 \mathrm{~mm})$, al que le sigue una etapa (1934-1939) de importante descenso $(8,51 \mathrm{~mm})$ y otra de estancamiento (1940-1945), para culminar al final del período con un incremento $(8,50 \mathrm{~mm})$ que permite que la serie alcance el mismo valor que tenía veinte años antes.

Por tanto, existen tres períodos de crecimiento, dos de ellos muy importantes (1894-1908 y 1929-1933) y un tercero (1940-1953) más modesto que logra que la estatura de los españoles recupere valores anteriores; dos etapas de estancamiento, una bastante prolongada (1909-1928) y otra más breve (1940-1945); y, por último, un período (1934-1939) de descenso de la estatura media que supone retroceder a valores de una década anterior.

La explicación de estas fluctuaciones en la serie de estatura se encuentra en los cambios de edad de reclutamiento, y muy especialmente en la situación socioeconómica derivada de los acontecimientos históricos que vivió España durante este período. Haciendo un ejercicio de comparación de nuestra serie de estatura con la de renta per cápita de Prados de la Escosura (Gráfico 2), se observa que el perfil de ambas es prácticamente idéntico, si se aplica un gap temporal a la serie de renta de cuatro años, es decir, cuando los reclutas contaban con dieciséis-diecisiete años (etapa de pleno crecimiento adolescente) ${ }^{20}$. Esta similitud de ambas series corrobora la hipótesis de que la estatura es un reflejo de las condiciones materiales de vida, uno de cuyos principales componentes, aunque no el único, es la renta.

Asimismo, existe una correlación casi perfecta entre la evolución de la serie de estatura y la de las tasas de supervivencia de cada cohorte de edad, lo que implica que la estatura es también un reflejo de las condiciones sanitarias ${ }^{21}$.

${ }_{19}$ Udjus (1964); Brinkman, Drukker y Slot (1988); Costanzo (1948).

20) Los datos de renta per cápita, en Prados de la Escosura (1995).

${ }^{21}$ Quiroga y Coll (2000). 


\section{GRÁFICO 2}

Estatura media de los reclutas españoles (1893-1954) y Renta per cápita al coste de los factores (1890-1949)

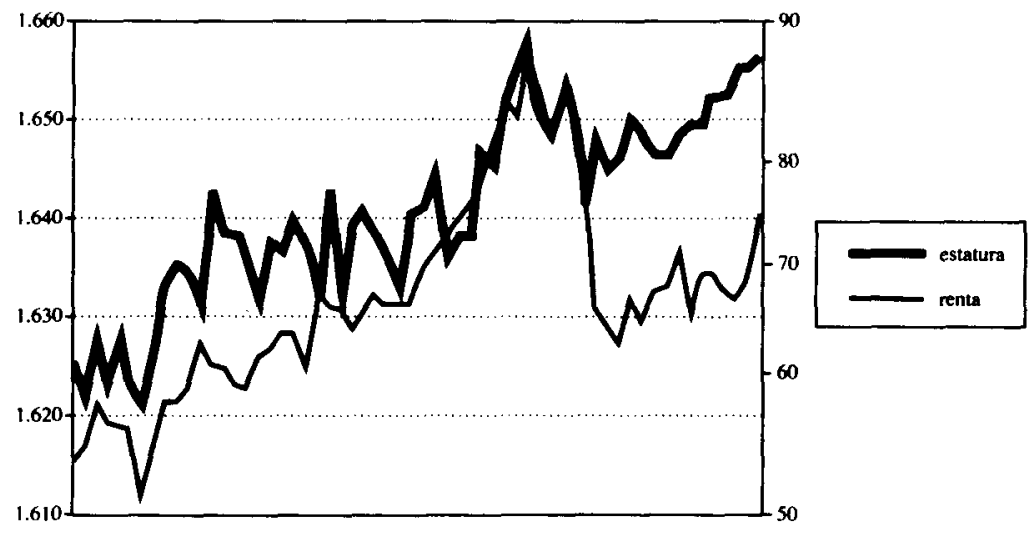

Fuentes: Archivo General Militar y Prados de la Escosura (1995).

$\mathrm{El}$ aumento de casi $1,5 \mathrm{~cm}$ que tuvo lugar durante la primera etapa (1894-1908) pudo deberse en parte a los sucesivos cambios en la edad de reclutamiento de los mozos españoles, que pasó de diecinueve a veintiún años. Sin embargo, el segundo período de gran aumento (1929-1933), parece que debemos atribuirlo a la favorable coyuntura socioeconómica (crecimiento económico, aumento de la renta y mayor igualdad en la distribución de la renta) de la segunda parte de la década de los veinte ${ }^{22}$.

El período de descenso de la estatura media (1934-1939) y el de estancamiento que le sigue (1940-1945) coincide con hechos cruciales en la historia de España: la depresión de los años treinta, el clima de enrarecimiento social, económico y político de los años de la II República, la Guerra Civil, la posguerra española y las consecuencias para España de los inicios de la II Guerra Mundial. También podría influir el hecho de que durante este período se asiste a un adelantamiento en la edad de reclutamiento; sin embargo, la reducción de las estaturas se observa también en los años en los que el reclutamiento se efectuó en la edad habitual, lo cual invita a pensar que hubo otras fuerzas actuando.

\footnotetext{
${ }^{22}$ Quiroga y Coll (2000).
} 
La última etapa (1946-1953), de tímida recuperación, refleja el inicio del lento camino hacia el restablecimiento de la situación prebélica. Esta recuperación contrasta con las estimaciones de la renta per cápita para el mismo período, en las que no se aprecia un gran aumento ${ }^{23}$. Todo ello parece indicar que a pesar de que la renta no creció, las condiciones materiales de vida de los españoles sí que mejoraron (probablemente por el consumo de bienes no intercambiables, mejoras en las condiciones sanitarias tras la Guerra Civil, etc.).

El diseño de la muestra permitió también construir series regionales, que muestran las importantes diferencias en las estaturas medias por regiones; estas diferencias se mantienen, a lo largo del tiempo, del orden de unos seis centímetros ${ }^{24}$.

De manera resumida, y sin intentar hacer un análisis pormenorizado de la evolución regional, todas las series muestran una notable coincidencia en determinados movimientos a corto y medio plazo, hecho que interpretamos como indicativo de una aceptable fiabilidad de las muestras regionales. Aunque en los comienzos de la serie la evolución es más dispar (quizás por la peor calidad del muestreo), la mayor parte de las regiones muestran una tendencia al alza - con las excepciones de Castilla y León y Extremadura, que se mantienen prácticamente estancadas a lo largo del período-, una depresión en la segunda parte de la década de los treinta y una recuperación al final del periodo.

Además, destaca el hecho de que algunas regiones muestran «saltos» muy localizados en el tiempo, que hacen que su estatura media varíe. Ejemplos destacados son el caso catalán, con dos fases de crecimiento: una menor, en la segunda parte de la década de 1910, y una muy importante entre 1924-1931; Castilla-La Mancha con otros dos momentos de crecimiento, uno entre 1910-1920 y otro entre 1926-1931; Madrid, a partir de 1910; Navarra, con un crecimiento espectacular entre 1918-1925; y Extremadura en la primera década del siglo $\mathrm{XX}^{25}$. También existen cambios de nivel importantes, pero de tendencia negativa, en Extremadura y Cantabria con la gran depresión que sufren a principios de siglo $\mathrm{xx}$; en el

${ }^{23}$ Prados de la Escosura (1995).

${ }^{24}$ Las representaciones gráficas de las series regionales y por categorías socioprofesionales se pueden ver en Quiroga (1998).

${ }^{25}$ Cataluña aumenta entre 1917-1920, 30,19 mm, y entre 1924-1931, 24,0 1mm; Castilla-La Mancha, entre 1910-1920, de 22,49 mm, y entre 1926-1931 de 23,09 mm; Madrid, entre 1909.1916, de 42.54 mm; Navarra, entre 1918-1925, de 48,10 mm, y Extremadura, entre 1903-1911, de 67,80 mm. 
País Vasco, entre 1910 y 1915; o en los años 1922-1926 y 1929-1933 en La Rioja ${ }^{26}$.

Mención especial merece el caso Cantabria, del que no se disponen datos entre 1905-1930. Su evolución es muy peculiar, ya que muestra una tendencia descendente desde 1893 que le hace situarse en 1904 en las posiciones más bajas dentro del conjunto nacional; entre esta fecha y 1930 experimenta un crecimiento de más de seis centímetros $(66,43 \mathrm{~mm})$, dos veces superior a la media nacional para todo el período. Este comportamiento de la estatura en Cantabria es muy acorde con la evolución económica de la región: un declive a finales del siglo XIX y un rápido crecimiento, propiciado principalmente por la especialización lechera y la aparición de núcleos industriales modernos, durante el cuarto de siglo siguiente ${ }^{27}$.

Analizando conjuntamente las tendencias a largo plazo de las series autonómicas existen regiones que mantienen constantemente una estatura baja, y regiones con una elevada estatura, mientras que otras ven cómo su lugar dentro del conjunto nacional cambia a lo largo del tiempo, lo que nos permitiría hablar de mayor o menor dinamismo regional (Cuadro 1) ${ }^{28}$.

A finales del siglo $\mathrm{xIx}$, las regiones con una menor estatura eran Castilla y León, Castilla-La Mancha, Extremadura y Galicia. La característica común de este grupo es que son regiones poco desarrolladas, aunque coexisten regiones agrícolas y secas, como son las dos Castillas y Extremadura, con Galicia caracterizada por un sistema de propiedad muy fragmentada. También destaca la escasa estatura de Madrid a comienzos del siglo xx; la explicación puede encontrarse en la escasa estatura de la mayor parte de sus inmigrantes, procedentes de ambas Castillas (los más «bajitos» del país) y en que era una gran urbe con todavía un medioambiente epidemiológico desfavorable.

En el otro extremo, los españoles más altos de fines del XIx vivían en Cataluña, el País Vasco y Canarias. Las dos primeras eran regiones industrializadas y Canarias es un archipiélago de clima semi-tropical con unas peculiaridades étnicas diferentes a las de la Península, ya que los ancestros de esta población, los guanches, que habitaron las Islas hasta el siglo XV, eran muy altos según muestran las evidencias arqueológicas ${ }^{29}$.

${ }^{26}$ Extremadura pierde entre 1896-1902, 29,81 mm; Cantabria, entre 1894-1903, 31,29 $\mathrm{mm}$; el País Vasco, entre 1909-1915, 27,07 mm, y La Rioja, entre 1922-1926, 28,98 mm y, entre 1929 y $1933,21,67 \mathrm{~mm}$.

${ }_{27}$ Domínguez y De la Puente (1997).

${ }^{28}$ Las tasas de crecimiento calculadas son exponenciales.

${ }^{29}$ Hace 2500 años presentaban una estatura de $1,70 \mathrm{~cm}$ para el caso masculino. 


\section{CUADRO 1}

Ranking autonómico en orden creciente de estaturas y tasas de crecimiento

\begin{tabular}{|c|c|c|c|c|}
\hline & $1893-1899$ & 19201925 & $1949-1954$ & $\begin{array}{c}\text { Tasa } \\
\text { de crecimiento } \\
1893-1954\end{array}$ \\
\hline Andalucía ........... & 9 & 7 & 2 & 0,03 \\
\hline 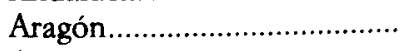 & 10 & 4 & 8 & 0,06 \\
\hline 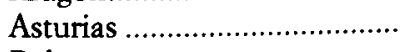 & - & - & 10 & - \\
\hline 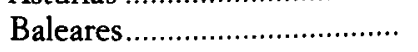 & 11 & 9 & 11 & 0,03 \\
\hline 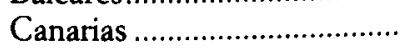 & 15 & 14 & 15 & 0,05 \\
\hline 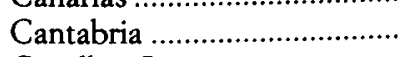 & 5 & - & 12 & 0,27 \\
\hline 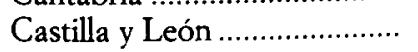 & 2 & 1 & 4 & 0,12 \\
\hline Castilla-La Mancha ................. & 1 & 5 & 5 & 0,07 \\
\hline 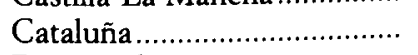 & 14 & 12 & 16 & 0,12 \\
\hline 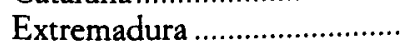 & 3 & 3 & 1 & 0,08 \\
\hline 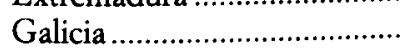 & 4 & 2 & 6 & 0,06 \\
\hline 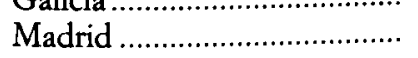 & 8 & 8 & 14 & 0,07 \\
\hline 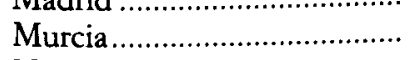 & - & - & 3 & 0,10 \\
\hline 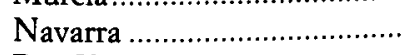 & 7 & 13 & 13 & - \\
\hline 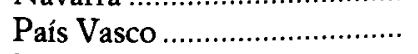 & 13 & 11 & 17 & 0,10 \\
\hline 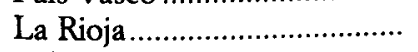 & 6 & 6 & 7 & 0,10 \\
\hline 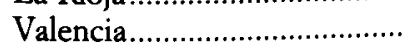 & 12 & 10 & 9 & 0,04 \\
\hline
\end{tabular}

FUENTE: Archivo General Militar.

Por último, sorprende la elevada posición dentro del ranking autonómico de Andalucía a finales del siglo XIX.

En 1954, las estaturas medias más bajas se situaban en Extremadura, Andalucía, Murcia y ambas Castillas, y las más altas en el País Vasco, Cataluña, Canarias y Madrid.

A lo largo de estos sesenta años, el orden de las regiones no varía considerablemente, salvo los casos de Madrid y Andalucía que experimentan grandes cambios de posición, como consecuencia de las transformaciones en sus condiciones de vida. Madrid consigue en la primera parte del siglo $\mathrm{xX}$ superar todos los obstáculos medioambientales y epidemiológicos que caracterizaban a las grandes ciudades decimonónicas y mejora sus condiciones sanitarias, lo que conllevaría un aumento de su estatura media ${ }^{30}$. Por el contrario, Andalucía, una región de gran dinamismo eco-

${ }^{30}$ Reher (2000). 
nómico a comienzos del siglo XIX, vio truncado su crecimiento en una época posterior, hecho que posiblemente esté detrás de la pérdida de posiciones relativas que refleja el Cuadro 1 .

En definitiva, y a la vista de los datos mostrados en el Cuadro 1, se observa la existencia de regiones que podríamos denominar estáticas, es decir, que mantienen un nivel similar a lo largo de todo el período, independientemente del lugar que ocupan en la clasificación; entre éstas destacarian Baleares, Canarias, Castilla y Léon, Cataluña, La Rioja y, en menor medida, Galicia (ya que presenta una mejora importante a comienzos del siglo $\mathrm{xx}$ ). Por otro lado, estarian las regiones dinámicas, que ven cómo su posición relativa varía durante estos sesenta años, tanto positivamente (es el caso de Cantabria, Castilla-La Mancha, Madrid, y Navarra) como negativamente (Andalucía, Valencia y, en menor medida, Extremadura y Aragón).

Además de los datos relativos a su origen geográfico, las Hojas de Filiación de cada recluta proporcionan información sobre la profesión de los individuos, lo que ha permitido realizar la clasificación de los reclutas por grupos socioprofesionales. La principal debilidad de esta clasificación es que estamos hablando de la profesión del recluta y no de la de sus progenitores, que probablemente fuera más importante a la hora de explicar el nivel de renta y las condiciones medioambientales del recluta. Sin embargo, era una práctica bastante habitual anotar en la Hoja de Filiación la profesión del padre, en lugar de la del recluta.

Como es de suponer, el abanico de profesiones era muy amplio. Las cerca de setecientas que aparecieron registradas en la muestra fueron agrupadas en nueve categorías, con el objetivo de agregar sectorialmente y por productividad del trabajo: propietarios agrícolas, trabajadores agrícolas, artesanos, trabajadores industriales, trabajadores de la construcción, trabajadores de servicios tradicionales (peluqueros, sastres, porteros, etc.), trabajadores de servicios modernos (ferroviarios, mecánicos, tipógrafos, etc.), empleados de cuello blanco, y profesionales y estudiantes).

En primer lugar, destacan dos hechos: la estabilidad de las categorías dentro del ranking (Cuadro 2) y la persistencia de diferencias entre determinadas series de estatura por profesiones de entre tres y cinco centímetros que, además, nunca convergen. No obstante, la evolución de las series es desigual: hay grupos que experimentan un incremento importante en su estatura media (empleados de cuello blanco con más de seis centímetros; estudiantes y profesionales con casi cinco; y trabajadores de servicios modernos e industriales con cuatro), y otros, como los trabajadores agrí- 


\section{CUADRO 2}

Ranking socioprofesional en orden creciente de estaturas y tasas de crecimiento

\begin{tabular}{|c|c|c|c|c|}
\hline & $1893-1899$ & $1920-1925$ & $1949-1954$ & $\begin{array}{c}\text { Tasa } \\
\text { de crecimiento } \\
1893-1954\end{array}$ \\
\hline Obrero agrícola ....................... & 1 & 1 & 1 & 0,08 \\
\hline Propietario agrícola ................ & 2 & 2 & 5 & 0,09 \\
\hline 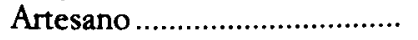 & 5 & 3 & 3 & 0,08 \\
\hline Obrero industrial ................... & 3 & 5 & 6 & 0,09 \\
\hline Servicios tradicionales........... & 4 & 4 & 2 & 0,10 \\
\hline Servicios modernos ................. & 8 & 8 & 7 & 0,09 \\
\hline Cuello blanco ........................ & 7 & 7 & 8 & 0,14 \\
\hline Profesional/Estudiante.......... & 9 & 9 & 9 & 0,09 \\
\hline Trabajador construcción ........ & 6 & 6 & 4 & 0,08 \\
\hline
\end{tabular}

FUENTE: Archivo General Militar.

colas, de servicios tradicionales y artesanos que sólo aumentan su estatura media en algo más de dos centímetros a lo largo de todo el período.

Mención aparte merece el caso de los propietarios agrícolas, que mantienen a lo largo del período una trayectoria muy similar a la de los obreros agrícolas, salvo en los últimos quince años, donde su estatura aumenta de manera importante mientras los obreros ven cómo la suya se estanca. Así, de los cuatro centímetros de aumento de esta categoría, dos se consiguen a partir de $1941^{31}$. Este aumento hace que la posición relativa de los propietarios mejore, tal y como muestra el Cuadro 2.

El grupo que muestra incrementos en sus estaturas está formado por aquellos cuya alimentación es mejor, bien por su posición social (profesionales y empleados de cuello blanco) o bien porque su nivel de ingresos se lo permitía al trabajar en actividades modernas (obreros industriales y servicios modernos).

Analizando por subperíodos, los empleados de cuello blanco experimentan el crecimiento más pronunciado en el periodo anterior a la Guerra Civil, a pesar del bache sufrido entre 1910-1915. En los años posteriores al conflicto bélico, el aumento se concentra en los profesionales y estudiantes, en los propietarios agrícolas y, nuevamente, en los empleados de cuello blanco. Finalmente, en la última etapa, la estatura de los trabajadores

${ }^{31}$ Quiroga (1997). 
agrícolas, de la construcción y de los servicios tradicionales no muestra aumento alguno.

Resumiendo, todo parece indicar que las profesiones que muestran los mayores aumentos en su estatura podrían haber sido las que experimentaron un incremento más rápido en su productividad (y en sus salarios) además de tener una menor estacionalidad en el empleo y de habitar en el mundo urbano, mientras que la estatura mejoró menos en aquellas actividades caracterizadas por un bajo crecimiento en su productividad ${ }^{32}$.

\section{EVOLUCIÓN TEMPORAL DE LAS DESIGUALDADES REGIONALES Y ENTRE GRUPOS SOCIOPROFESIONALES DE LA ESTATURA}

Partiendo de las series de estatura media por regiones y por categorías socioprofesionales, se puede tratar de profundizar en el tema de las desigualdades regionales y de otro tipo en cuanto a condiciones materiales de vida y a su evolución a lo largo del tiempo. Enlazando con el tema sobre la convergencia entre países y/o regiones, se trata de ver qué puede aportar al debate el estudio de las estaturas. De manera resumida se trataría de averiguar si, durante el período analizado, se produjeron procesos de convergencia y/o divergencia en las condiciones materiales de vida entre regiones y entre grupos socioprofesionales, y, en caso afirmativo, qué fenómenos pueden explicar tales procesos.

Para obtener la primera de esas medidas, en cada año se ha calculado la desviación típica de las medias anuales provinciales, para obtener una serie temporal del coeficiente de variación. La segunda de las medidas calculadas es el coeficiente de variación de las medias correspondientes a los diferentes grupos socioprofesionales, cuya serie temporal se ha obtenido de manera análoga a la anterior. Además de los valores anuales, hemos calculado medias móviles de nueve años de este coeficiente de variación con el fin de evitar los movimientos erráticos imputables al error de muestreo que afecta a las medias provinciales, de suavizar su evolución en el corto plazo y de constatar la existencia de etapas diferenciadas.

El Gráfico 3 muestra una tendencia prácticamente plana, lo que prueba que, entre finales del siglo xIX y la primera mitad del $\mathrm{Xx}$, la desigualdad regional de las estaturas se mantuvo estable. Esta tendencia plana del coe-

${ }^{32}$ Reher (2000). 


\section{GRÁFICO 3}

Coeficientes de variación provinciales (1893-1954)

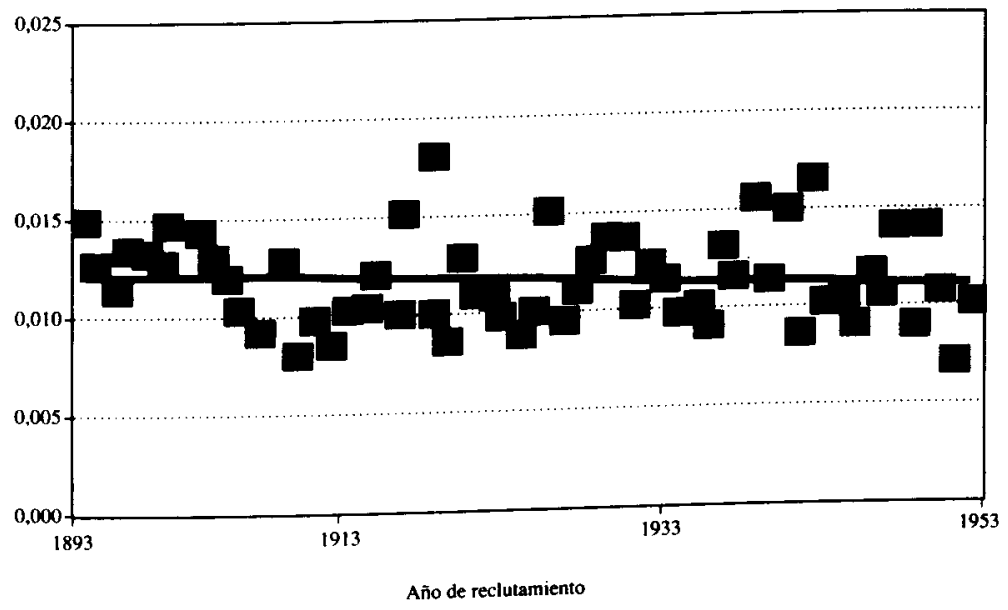

FuENTE: Archivo General Militar.

\section{GRÁFICO 4}

Coeficientes de variación provinciales.

Medias móviles en nueve años (1897-1950)

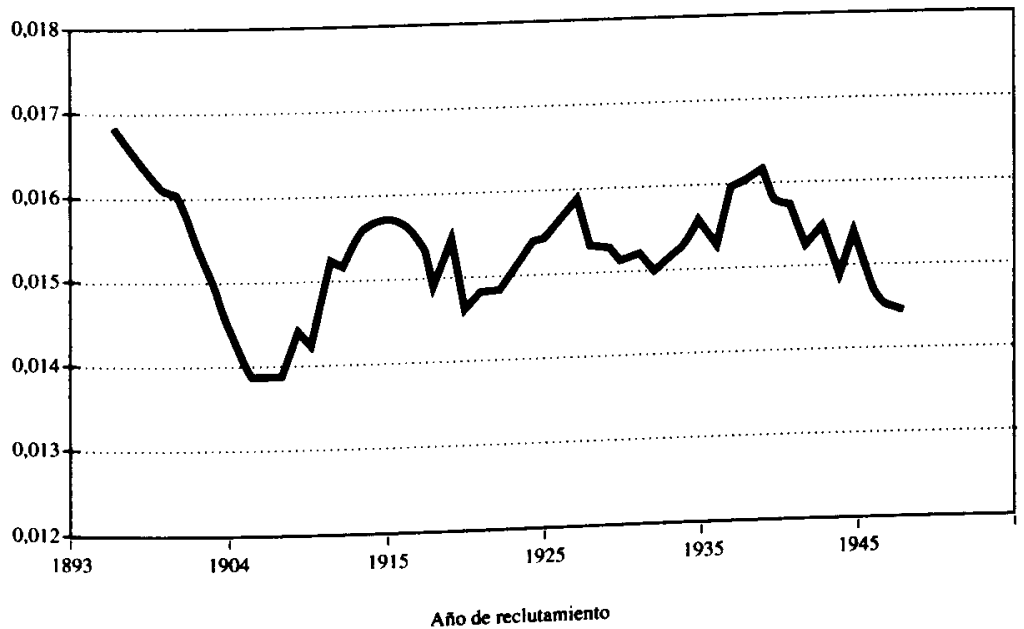

FuENTE: Archivo General Militar. 
ficiente de variación, unida a la no existencia de ciclos largos, queda corroborada por la ausencia de correlación entre las posiciones de las regiones españolas dentro del ranking español a comienzos del período y las tasas de crecimiento de cada una de ellas (Cuadro 1).

A la vista de todo ello, parece acertado concluir que estamos ante una ausencia de convergencia o divergencia regional. La posible explicación a este comportamiento reside en que los movimientos migratorios interregionales consiguieron equilibrar las condiciones materiales de vida de las diferentes regiones españolas ${ }^{33}$. Obviamente, si estos movimientos no hubieran existido, el resultado de este coeficiente de variación habría sido muy diferente ${ }^{34}$.

Se ha repetido el mismo ejercicio para el caso de las estaturas por categorías socioprofesionales (Gráficos 5 y 6), con el resultado de que el coeficiente de variación de las estaturas por profesiones presenta una tendencia ascendente. Ello parece indicar que, entre finales del siglo $\mathrm{xIX}$ y la primera mitad del $\mathrm{xx}$, aumentaron las desigualdades en cuanto a condiciones materiales de vida de los diferentes estratos de la sociedad. Además, el coeficiente de variación de las categorías socioprofesionales muestran cuatro fases bien diferenciadas ${ }^{35}$ (Gráfico 6).

De manera resumida, los datos apuntan una evolución diferente de la desigualdad de las estaturas por provincias y por categorías socioprofesionales. Si la evolución de la desigualdad de las estaturas es un reflejo de la desigualdad en las condiciones materiales de vida, ¿es consistente la presencia de movimientos de convergencia/divergencia entre grupos socioprofesionales y la ausencia de movimientos similares entre regiones? La respuesta es, en nuestra opinión, afirmativa.

Según el enfoque neoclásico desarrollado en la teoría del crecimiento, los países tienden a la convergencia ${ }^{36}$. Sin embargo, esta caracterización de la teoría neoclásica del crecimiento observable no lo es en la realidad.

${ }^{33}$ Quiroga (2001). Los datos muestran que aquellos reclutas que emigraron desde regiones más atrasadas hacia otras más desarrolladas presentan una estatura más elevada que los que permanecieron en sus regiones de origen.

${ }^{34}$ Quiroga (2001). La simulación ha sido realizada considerando que no existen movimientos migratorios interregionales, es decir, utilizando la provincia de nacimiento en lugar de la de reclutamiento.

3s Sobre este punto, véase el trabajo de Quiroga y Coll (2000).

${ }^{36}$ La explicación última reside en que los rendimientos del capital son decrecientes en el margen. Es decir, cualquier incremento de igual magnitud en el stock de capital por trabajador, tendría más efecto en los países o regiones atrasadas, por lo que éstos tenderían a converger en niveles de renta con los más desarrollados. Para un buen estado de la cuestión, véase Aghion, Caroli y García Peñalosa (1999). 


\section{GRÁFICO 5}

Coeficientes de variación de categorias socioprofesionales (1893-1954)

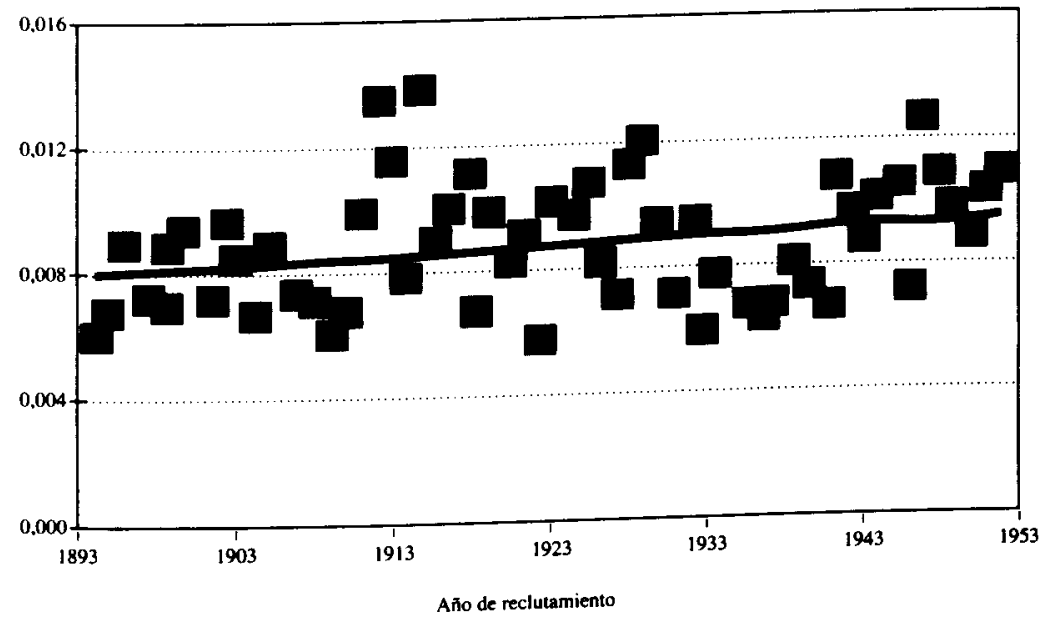

FUENTE: Archivo General Militar.

No obstante, existe otra corriente en economía que sí se fija en las diferencias sectoriales de productividad, estudiando las tendencias en la distribución personal de la renta y la evolución en el tiempo de éstas en los períodos de crecimiento económico (resumida en la famosa $U$ invertida de Kuznets) ${ }^{37}$. Incluso hay autores que defienden que precisamente las diferencias en la productividad del factor trabajo entre las diversas actividades económicas son las que explican la desigualdad en el ingreso ${ }^{38}$. Obviamente, en la medida en que las actividades económicas modernas presenten una concentración regional, las tendencias en la distribución personal de la renta pueden tener un reflejo en la desigualdad de renta entre regiones. No obstante, existen otras fuerzas, tales como la emigración, que pueden debilitar este reflejo, colaborando en la igualación de la renta geográficamente.

Así pues, en los inicios de un proceso de modernización económica se produce una clara divergencia sectorial de renta, motivada por el aumento de productividad ( $y$, por ende, de salarios) de las profesiones del sector

${ }^{37}$ Véase Kuznets (1955).

${ }^{3 *}$ Por ejemplo, Williamson (1985). 


\section{GRÁFICO 6}

Coeficientes de variación de categorias socioprofesionales.

Medias móviles de nueve años (1897-1950)

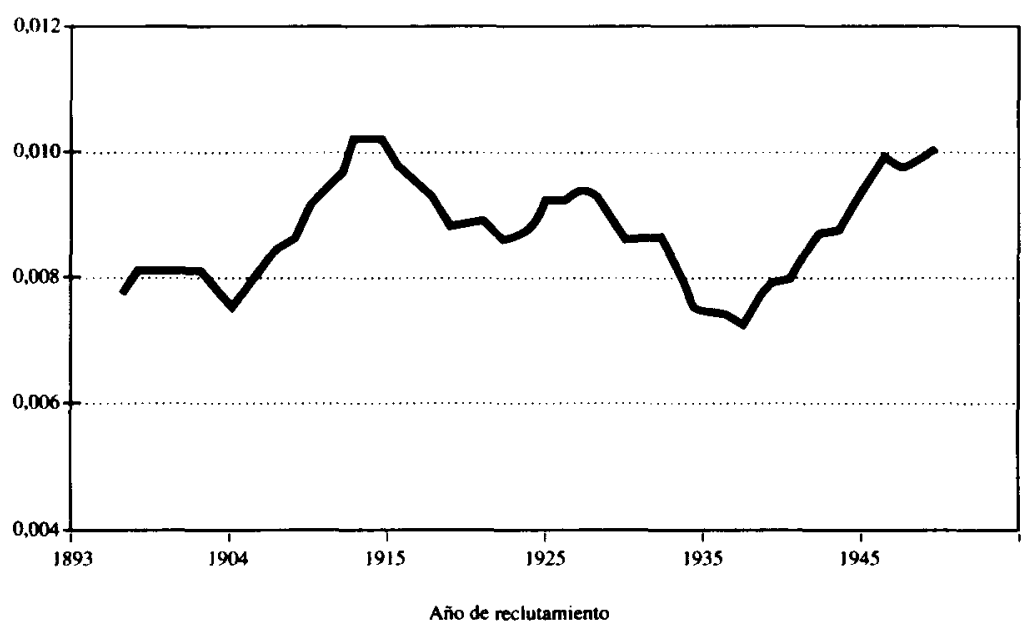

FUENTE: Archivo General Militar.

moderno, pero no obligatoriamente una divergencia espacial, ya que ésta sólo se dará si el sector moderno se concentra en determinadas regiones o si no existen otras fuerzas (como los movimientos migratorios) que permitan la igualación espacial de rentas.

Por tanto, la principal conclusión es que pueden existir procesos simultáneos de convergencia y/o divergencia entre sectores y entre segmentos de la sociedad, y, simultáneamente, ausencia de procesos paralelos entre regiones (tal y como muestran los coeficientes de variación de las estaturas a nivel provincial y socioprofesional).

Hasta este momento toda la atención se ha puesto en la renta o en el producto interior bruto per cápita para comprender la posible evolución divergente de la desigualdad sectorial y espacial. Sin embargo, si pasamos a hablar de las condiciones materiales de vida, que es lo que refleja la estatura, a todos los motivos anteriores que contribuían a explicar la no existencia de procesos simultáneos entre regiones y sectores, se le suman otros, como pueden ser la existencia de diferencias en las condiciones sanitarias, en las tasas de fecundidad, entre barrios, entre campo y ciudad, etc., que no supongan diferencias similares entre regiones. 


\section{GRÁFICO 7}

Estatura media de los reclutas españoles por lugar de residencia (1893-1954). Medias móviles trienales

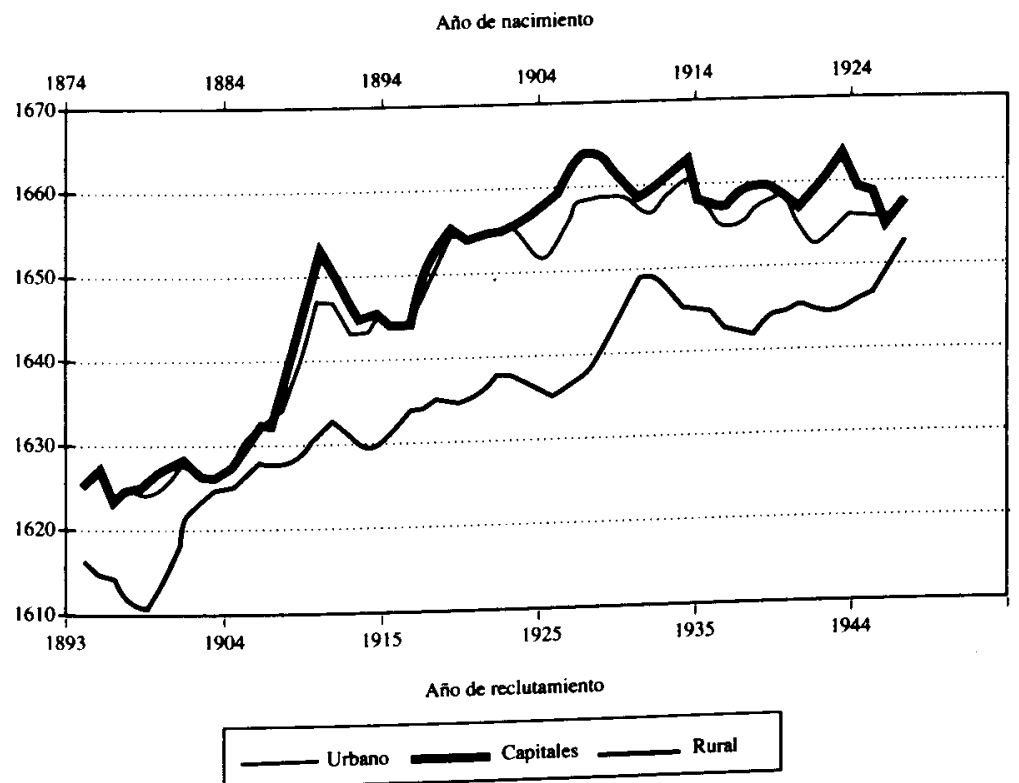

FUENTE: Archivo General Militar.

Esta última puntualización tiene su reflejo en la evolución de la estatura media de los reclutas españoles por lugar de residencia (urbano/rural) que muestra diferencias entre ambas categorías, menores al principio y al final del período, pero importantes a lo largo de la primera mitad del siglo $\mathrm{xx}^{39}$. (Gráfico 7). Estas diferencias se agudizan si, en lugar de considerar el mundo urbano como la suma de capitales de provincias y ciudades, atendemos exclusivamente a las capitales, donde por lo menos, en el siglo $\mathrm{xx}$, el acceso a la sanidad y las condiciones de salubridad eran mayores ${ }^{40}$.

${ }^{39}$ La clasificación de zona urbana incluye a las capitales de provincia, así como a los núcleos mayores de 10.000 habitantes hasta 1910 y más de 20.000 desde 1920 , según los Censos de la Población Española.

40 Reher (2000) identifica el paso de la «penalización» a la «ventaja» urbana en el caso español hacia 1930, unas fechas ligeramente más tardías que en el caso de las estaturas. Sin embargo, afirma que en el mundo urbano se obtenían salarios mayores y una menor 
Lo más destacable de los gráficos es que en dos momentos las series tienden a tocarse, entre $1905-1910$ y a partir de 1950 , mientras que en el resto del período, las diferencias entre ambas son grandes. La explicación a esta evolución pudiera residir tanto en las desigualdades sectoriales (concentración de actividades más productivas en el mundo urbano) como en las mejoras producidas en las condiciones sanitarias, que obviamente afectaron en primer lugar a los centros urbanos, especialmente a las capitales de provincia. En definitiva, lo que indican estos gráficos es la existencia de movimientos de convergencia y divergencia, en cuanto a condiciones materiales de vida, entre territorios diferentes - como son campo y ciudad-, que no tienen, sin embargo, un paralelo cuando tomamos como unidad a las regiones.

\section{CONCLUSIONES}

A lo largo de este artículo se ha intentado mostrar cómo la estatura puede ser utilizada como un indicador alternativo de mediciones de bienestar. Aunque por todas sus deficiencias no puede suplantar a las variables de las cuentas nacionales, sí que puede ser utilizada como alternativa o complemento para mejorar las deficiencias de medición del producto nacional como medida de bienestar o para conocer la calidad de vida en períodos o en grupos para los cuales las medidas convencionales no pueden ser calculadas.

Precisamente el caso español presenta estas deficiencias, y por ello el uso de indicadores alternativos resulta muy útil. La riqueza de información antropométrica es considerable y sus principales ventajas serían: la universalidad masculina - ya que existen datos de estatura para la práctica totalidad de los varones españoles (excepto los prófugos, los que hicieron la carrera militar y los que cumplieron su servicio militar en el Ejército del Aire y en la Marina)—; la posibilidad de construir, entre otras, series regionales, socioprofesionales y por lugar de residencia que permiten interesantes comparaciones, y el hecho de proporcionar información sobre muchos aspectos diferentes a los habituales (es decir, nutrición y condiciones sanitarias), tales como productividad del factor trabajo, movilidad interregional, niveles educativos, relación entre estatura y tasas de mor-

estacionalidad en el empleo, lo que permitía unos niveles nutricionales mayores, que, junto a unos niveles de vida mejores, favorecieron una mayor resistencia a la enfermedad. 
talidad y/o de supervivencia, y sobre la desigualdad en los niveles de bienestar.

Los resultados expuestos muestran, en primer lugar, la evolución de la estatura media de los reclutas españoles a nivel nacional, regional y socioprofesional. Sugieren una estrecha relación entre la estatura, la renta y las condiciones medioambientales, y corroboran que la estatura es un buen indicador de las condiciones materiales de vida. En segundo lugar, muestran dos experiencias distintas entre la desigualdad de la estatura por regiones y por grupos socioprofesionales, quedando clara la trayectoria ascendente de la desigualdad personal (que, además, muestra unas fases muy diferenciadas) y no tan obvia la de la desigualdad regional.

No obstante, la reconciliación entre ambos comportamientos queda explicada a la luz de la teoría neoclásica que identifica a las diferentes tasas del crecimiento de la productividad por sectores como el principal factor a la hora de explicar las tendencias en la distribución personal del ingreso (o en nuestro caso, en las condiciones materiales de vida). Por lo que respecta a las desigualdades regionales, los resultados parecen corroborar las conclusiones latentes en todos los enfoques basados en la función de producción agregada. Aunque de este trabajo no se obtiene ninguna conclusión con respecto a la convergencia o divergencia entre regiones, la ausencia de tendencia en este terreno contrasta con el patrón, más claro, que se observa al comparar grupos socioprofesionales. Eso parece indicar que, en efecto, la convergencia o divergencia entre regiones parece depender más de fenómenos agregados que de las diferencias de productividades entre sectores.

\section{BIBLIOGRAFÍA}

Aghion, P.; Caroli, E., y García Peñalosa, C. (1999): «Inequality and Economic Growth: The Perspective of the New Growth Theoriesw, Joumal of Economic Literature, 37, pp. 1615-1660.

Archivo General Milttar, Sección Tropa. Guadalajara.

Brinkman, H. J.; DrukKer, J. W., y Slor, B. (1988): «Height and Income: A New Method for the Estimation of Historical National Income Series», Explorations in Economic History, 25, 3, pp. 227-264.

CIPOLla, C. (1992): Historia Económica de la Europa preindustrial, Madrid, Alianza. Coll, S., y Komlos, J. (1998): «The Biological Standard of Living and Economic Development: Nutrition, Health and Well Being in Historical Perspective», en C. E. NúNEz (ed.), Debates and Cotroversies in Economic History. Prooceedings 
of Twelfth International Economic History Congress, Madrid, Centro de Estudios Ramón Areces, pp. 219-282.

Coll, S., y Quiroga, G. (2000): The Biological Standard of Living Under Franco Dictatorship, Paper presentado al Third European Social Science History Conference, Amsterdam, 12-15 de abril de 2000.

Colección Legislattva del Ejército (1893-1999), Madrid, Ministerio de Defensa. Costanzo, A. (1948): «La statura degli italiani ventenni nati dal 1854 al 1920», Annali di Statistica, Serie 8, 2, pp. 63-123.

Dominguez, R., y De la Puente, L. (1997): «Dependencia de la trayectoria y cambio técnico en la ganadería de Cantabria, 1750-1930», en S. LOPEZ y J. M. VALDALISO (eds.), ¿Que inventen ellos? Tecnologia, empresa y cambio técnico en la España contemporánea, Madrid, Alianza, pp. 121-155.

Estadística de Reclutamiento y Reemplazo (1911-1929, 1954-1999), Madrid, Ministerio de Defensa.

EveleTH, P. B. (1985): «Nutritional Implications of Differences in Adolescent Growth and Maturation and in Adult Body Size», en C. BLAXTER y J. C. Water. Low (eds.), Nutritional Adaptation in Man, London, Jonh Cibbey, pp. 31.43.

Eveleth, P. B., y Tanner, J. M. (1979): Worldwide Variations in Human Growth, Cambridge, Cambridge University Press.

Figuerola, L. (?): «La talla de los mozos para el Servicio Militar sorteados y medidos en las quintas de 1858 a 1867", Separata de Memorias de la Real Academia de las Ciencias Morales y Políticas, 7.

Floud, R.; Watcher, K., y Gregory, A. S. (1990): Heights, Health and History: Nutritional Status in UK, London, Cambridge University Press.

Fogel, R. W., y ENGERMAN, S. (eds.) (1982): Secular Trends in Nutrition, Labor Welfare and Labor Productivity, número especial de Social Science History, 6.

Galton, F. (1886/1887): «On Recent Designs for Anthropometric Instruments», Journal of the Antbropological Institute of Great Britain and Ireland, 16, pp. 2-8.

Gomez Mendoza, A., y Pérez Moreda, V. (1985): «Estatura y nivel de vida en la España del primer tercio del siglo XX», Moneda y Crédito, 174, pp. 29-64.

InStTTUTO Nacional de Estadística (varios años): Censo de la población española, Madrid.

KomLOS, J. (1989): Nutrition and Economic development in the 18th Century Habsburg Monarcby. An Anthropometric History, Princeton, Princeton University Press.

- (1994) (ed.): Stature, Living Standard and Economic Development. Essays in Anthropometric History, Chicago, Chicago University Press.

- (1995) (ed.): The Biological Standard of Living on Three Continents, Oxford, Westview Press.

KuzNETS, S. (1955): «Economic Growth and Income Inequality», American Economic Review, 45, pp. 1-28.

Martinez Carrión, J. M. (1985): «Estatura, nutrición y nivel de vida en Murcia, 1860-1930», Revista de Historia Económica, 4, 1, pp. 67-97.

- (1994): «Stature, Welfare and Economic Growth in 19th Century Spain: The Case of Murcia», en J. Komlos (ed.), Stature, Living Standard and Economic 
Development. Essays in Antbropometric History, Chicago, Chicago University Press, pp. 76-92.

- (1995): «Niveles de vida y desarrollo económico en la España contemporánea: una visión antropométrica», Revista de Historia Económica, 12, 3, pp. 685-718.

martínez Carrión, J. M., y Pérez Castejón, J. J. (1998): «Height and Standards of Living during the Industrialization of Spain: The Case of Elche», European Review of Economic History, 2, 2, pp. 201-230.

NICHOLAS, S., y STECKEL, R. (1991): «Heights and Living Standards of English Workers during the Early Years of Industrialization, 1770-1815», Journal of Economic History, 51, 4, pp. 937-957.

Nordhaus, D. N., y ToBin, J. (1973): «Is Growth Obsolete?», en M. Moss (ed.), The Measurement of Economic and Social Performance, New York, National Bureau of Economic Research, pp. 509-532.

OfFER, A. (2000): Economic Welfare Measurements and Human Well-Being, Paper presentado al Seminario del Departamento de Historia Económica de la Universidad Carlos III.

Oloriz, F. (1896): Discursos leidos en la Real Academia de Medicina para la recepción pública del académico electo... el día 24-5-96, Madrid, Imprenta y Librería de Nicolás Moya.

Prados de la Escosura, L. (1995): «Spain's Gross Domestic Product, 1850-1990: a new series», Madrid, Ministerio de Economía y Hacienda.

QueTelet, A. (1842): $A$ Treatise on Man and Development of His Faculties, New York, Burt Franklin.

- (1871): Anthropometrie ou mesure des differences facultes de l'bomme, Bruselas, C. Muguard.

QuiroGA, G. (1997): «Estatura y Niveles de vida en el mundo rural en España (1893-1954)», en Actas del VII Congreso de Historia Agraria, Salamanca, Universidad de Salamanca, pp. 189-204.

- (1998): «Height Evolution in Spain, 1893-1954: An Analysis by Regions and Professions», en J. Komlos y J. BATEN (eds.), Studies on Biological Standard of Living in Comparative Perspective, Stuttgart, Franz Steiner Verlag, pp. 359-383.

- (2001): La evolución de las medidas antropométricas como indicador del nivel de vida: Una aplicación a España (1893-1954), Tesis doctoral en curso de realización.

Quiroga, G., y Coll, S. (2000): «Height Inequality as a Proxy for Income Inequality. The Spanish case, 1895-1950», Joumal of Income Distribution, 9, pp. 107-131.

REHER, D. (2000): «In search of the urban penalty. Exploring urban and rural mortality patterns in Spain during the Demographic Transition», Paper presentando a la Social Science History Association, Pittsburgh, Pennsylvania, 26-29 de octubre.

SANDBERG, L., y STECKEL, R. H. (1980): «Soldier, Soldier, What Made you Grow so Tall? A Study on Height, Health and Nutrition in Sweden, 1720-1881», Economy and History, 23, 2, pp. 91-105. 
STECKEL, R. (1992): «Stature and Living Standards in the US», en R. GALLMAN y J. Wallis (eds.), American Economic Growth and Standards of Living before the Civil War, Chicago, University Press of Chicago, pp. 265-308.

- (1995): «Stature and the Standard of living», Journal of Economic Literature, 33, 4, pp. 1903-1940.

Udjus, L. G. (1964): Antbropometrical Changes in Norwegian Men in the 20th Century, Oslo, Universilelsforlaget.

Villermé, L. R. (1829): «Memoire sur la taille de l'homme en France», Annals d'Hygiene Publique et de Medicine Legale, 1, 2." parte, pp. 351-399.

WIERINGEN VAN, J. C. (1978): «Secular Growth Changes», en F. FALKNER y J. M. Tanner (eds.), Human Growth, 3 vols., vol. 3, New York, Plentum Press, pp. 445.473.

Williamson, J. G. (1985): Did British Capitalism Breed Inequality?, London, Allen\&Unwin. 